\title{
Canine Bite-Mark Evidence in Veterinary Necropsy: Case Studies Featuring the Bite-Mark Examination
}

\author{
Adrian Florin $\mathrm{GAL}^{1}{ }^{*}$, Roxana CORA ${ }^{1}$, Flaviu TĂBĂRAN ${ }^{1}$, Andras NAGY ${ }^{1}$, Cornel CĂTOI ${ }^{1}$ \\ Department of Pathologic Anatomy and Necropsy, Faculty of Veterinary Medicine, University of \\ Agricultural Sciences and Veterinary Medicine Cluj-Napoca, Calea Mănăștur 3-5, Cluj-Napoca, 400372, \\ Romania \\ *corresponding author, e-mail: adrian.gal@usamvcluj.므
}

Bulletin UASVM Veterinary Medicine 76(1)/2019

Print ISSN 1843-5270; Electronic ISSN 1843-5378

doi:10.15835/buasvmcn-vm: 2018.0046

\begin{abstract}
Forensic veterinary medicine tends to gain increasing importance in veterinary pathology, the number of such cases being still limited. The paper describes cases with multiple cutaneous wounds, with emphasis on bitemark analysis. The material was represented by corpses (deer and dogs) brought by local authorities to Faculty of Veterinary Medicine Cluj-Napoca (Romania), along with preliminary reports issued by the competent organs. A complete medicolegal survey was done. The cases analyzed by us displayed numerous usually symmetrical (opposed) wounds, with angular margins and elongated aspect (somehow specific to bite wounds). All cases assessed presented laceration of the skin from the subcutaneous tissue, rupture of muscle and (in some cases) perforation of internal organs. The features of the bite-marks described were typical for carnivores. Depending on the species involved, the topography of lesions varied. In deer, the ventrolateral area of the neck and the lumbar/ dorsal region were more commonly affected. The distribution suggests the hunting instinct of carnivores by trying to induce suffocation of the prey. In the analyzed dog corpses, the most affected areas were the auricular, limbs, sternal region, abdominal region. Investigation of bite-marks is an evolving field of forensic veterinary science and remains a provocation for veterinary pathologists.
\end{abstract}

Keywords: dog, deer, forensic, skin, bite-mark.

\section{Introduction}

Forensic veterinary medicine (FVM) is a medical field aimed to provide scientific medical evidence to the court of law. FVM tends to gain increasing importance in veterinary pathology, but the number of animal cases described (in various circumstances) is still in a limited number. The veterinarian specialist who performs the necropsy has to elaborate unbiased reports. Accordingly, the forensic medical practitioner, in addition to good professional training, must act impartially and in accordance with professional ethics, thus avoiding any medical error that might jeopardize the act of justice (Munro and Munro, 2008).
The paper describes a number of cases with multiple cutaneous wounds, with emphasis on bitemark analysis. The cases described were brought by the competent authorities to the Department of Pathological Anatomy, Necropsy Diagnosis and Forensic Medicine (Faculty of Veterinary Medicine Cluj-Napoca, Romania).

\section{Materials and methods}

The material was represented by corpses (deer and dogs) brought by local authorities to the Faculty of Veterinary Medicine Cluj-Napoca (Romania), along with preliminary reports issued by the competent organs. A complete medicolegal survey was done, including radiology exam of 
the carcass to exclude firearms wounds. For reasons of confidentiality, no personal details or circumstances of the facts committed in the analyzed cases will be revealed in the subsequent case presentations.

\section{Case no. 1.}

Examined subject and case history: Capreolus capreolus (European roe deer), female, found dead in the wild environment (probably killed by dogs); no witnesses. Queries stated in the preliminary report issued by the competent organs: determination of cause(s) that could generate the wounds and holes, including their features; the cause of death.

The external examination of the body concerned reveals numerous bite wounds (all with angular/elongated aspect), specific to bite wounds, most likely induced by dog or dogs (Figures. 1-6), all produced intra vitam in the following areas:

- right side of the body: skin wounds in the ventrolateral region of the neck $(2-2.5 / 1.5-2$ $\mathrm{cm})$, in the middle third of the thorax $(3 / 2 \mathrm{~cm})$, in the right lumbar region $(0.5 / 2 \mathrm{~cm}$;); a large penetrating wound $(5 / 6 \mathrm{~cm})$ in the thorax, with associated fracture of the ribs (ribs no. 11 to 13; Figure 2);

- left side: skin wounds in the cervicolateral (2.5/1 $\mathrm{cm}$; Figure 1), upper third of the thorax $(1.3 / 2.4$ $\mathrm{cm})$ and thigh regions $(4 / 2.5 \mathrm{~cm})$.

In all the aforementioned areas, the subcutaneous connective tissue and adjacent muscles undergone laceration, and associated diffuse bruises (including suggillations and hematomas).

The internal examination of the body revealed several penetrating wounds in the ruminal wall (Figure 5), in the middle third of the trachea (associated with hemorrhages in the tracheal mucosa and diffuse hemorrhages in the mediastinum; Figure 6), and rupture of the right diaphragmatic lobe of the lung. Other internal lesions identified were: pneumothorax, lung atelectasis, pulmonary edema, multiple hemorrhages in the lung parenchyma (bilaterally), and linear subendocardial ecchymoses. The death occurred because of polytrauma resulting from bite, the multiple penetrating wounds leading to respiratory failure (due to rib fractures and associated pneumothorax). No bullets (or metallic fragments) were found throughout the body following radiography and necropsy exams.
Concluding, the analyzed corpse of the deer presented several biting wounds localized mainly on the dorsal part of the body and in the ventral cervical region. According to the main features of the wounds and their distribution on the body, these were probably produced by a large animal, most likely dog/dogs.

\section{Case no. 2}

Examined subject and case history: Capreolus capreolus (European roe deer), male, found dead in the wild environment, with post-mortal changes; no witnesses. Queries stated in the preliminary report issued by the competent organs: identify the lesions and their features, and the cause of death.

The external examination of the body revealed bite wounds in the following regions: in the posterior upper-third third of the right hemithorax, wound size 2/1 cm with angled aspect; wound similar to the one previously described in the distal third of the neck, dorsally.

The internal exam of the body identified diffuse hemorrhages in the cervicoventral region (periesophageal and peritracheal) with the spread of hemorrhage in the mediastinum, without visible skin tearing. Other lesions observed were: diffuse pulmonary hemorrhages, pulmonary edema and multifocal capsular lung fibrosis in diaphragmatic lobes; granulomatous parasitic cellulitis in the dorsolumbar region (infection with Hipoderma spp.); parasitic granulomatous myocarditis (cysticercosis).

Concluding, the analyzed deer displayed multiple traumatic lesions with a dorsal and cervicoventral distribution that were induced most likely by biting. The aspect and distribution of the wounds and the cervicoventral traumas (that led to death of the deer by suffocation) are features someway specific to canine attacks.

\section{Case no. 3}

Examined subject and case history: dog body found in the wild environment, 6-year-old male, identified by microcipation, in good condition; the body was brought to perform the medicolegal exam due to suspicion of death by biting made by two other dogs (according to some witnesses statement).

The external examination of the body identified the following: gingival and conjunctival con- 


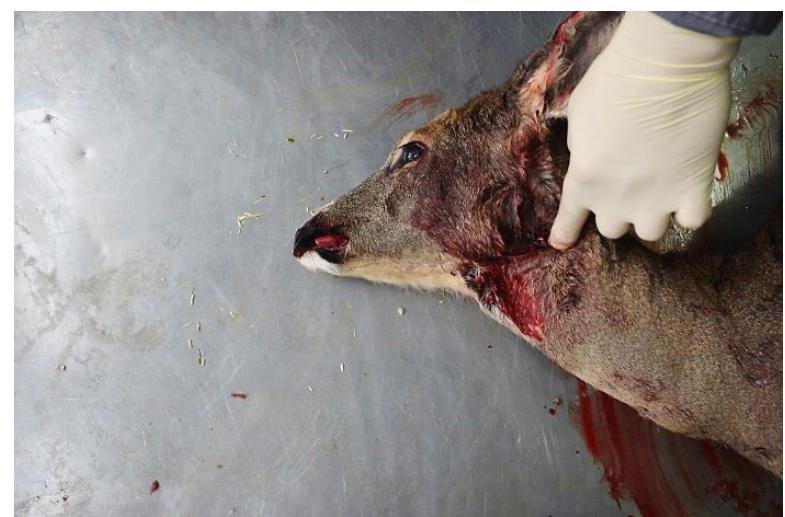

Figure 1. Bite wounds on the left ventrolateral side of the neck (roe deer killed by dogs).

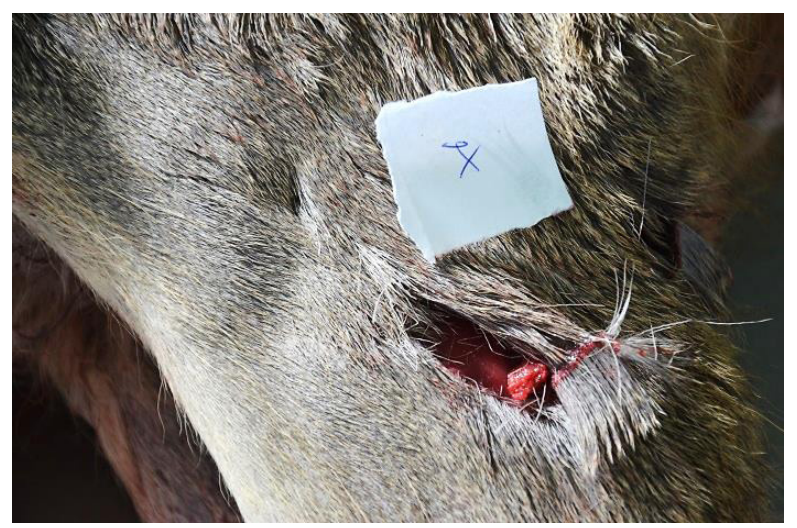

Figure 3. Angular aspect of the wound (neck ventral side).

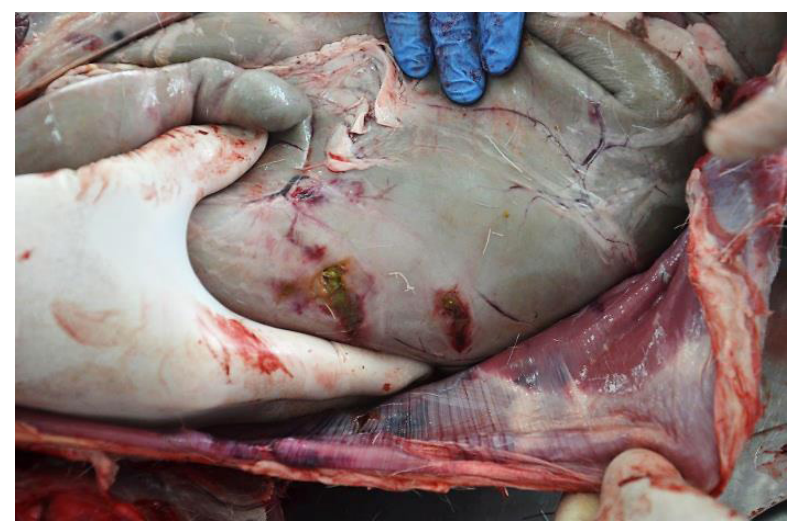

Figure 5. Perforation of the rumen following canines biting.

gestion; the presence of coagulated blood in the hair coat from several regions (chest, abdominal and dorsal regions, legs and around the muzzle); multiple, irregularly shaped and hemorrhagically infiltrated cutaneous wounds 0.5 to $5 \mathrm{~cm}$ in

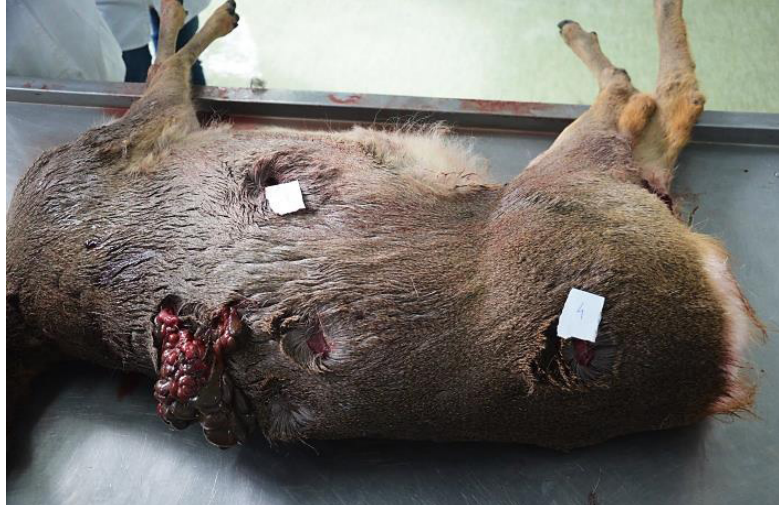

Figure 2. Distribution of bite wounds in the middle third of the chest and on the dorsal side and right lumbar region (roe deer killed by dogs).

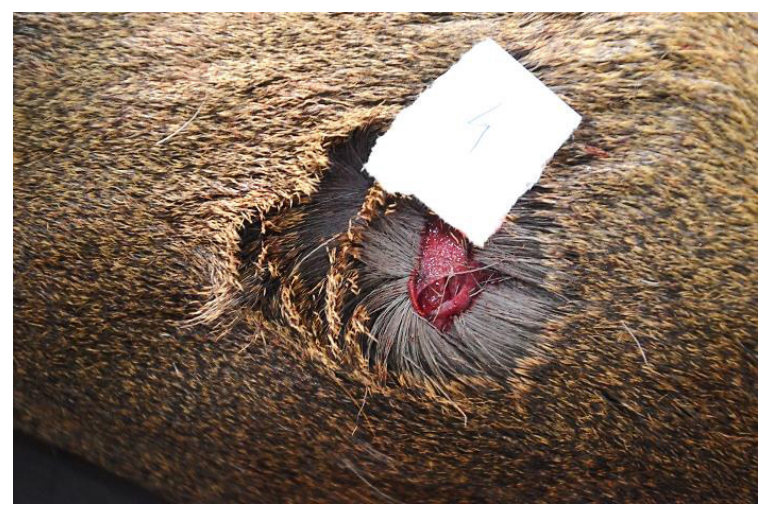

Figure 4. Bite wound aspect with blood infiltration on the wound margins (lumbar region detail).

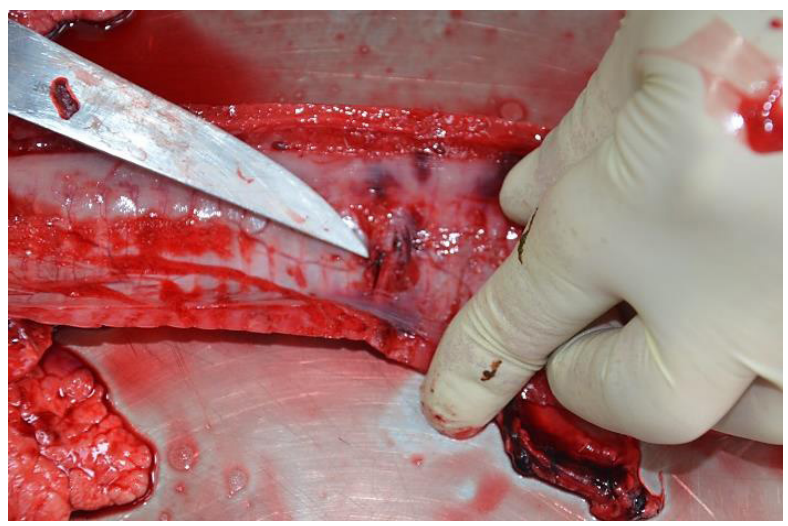

Figure 6. Biting wounds in the middle third of the trachea (associated with hemorrhages in the tracheal mucosa).

diameter), with the following distribution: left auricular area (base of the pinnae), right and left anterior legs (scapular region), right posterior leg (thigh and paralumbar regions), sternum, right flank, and ventral abdominal and inguinal regions. 
Multiple hemorrhages and hematomas (0.3$10 \mathrm{~cm}$ in size) were found in the subcutaneous tissue and subjacent muscles, with the following distribution: head (dorsal), neck (dorsal and lateral), thorax (dorsal and lateral), anterior left leg, abdominal and inguinal regions, lumbar region, and right posterior leg (lateral side). In the regions described, the subcutaneous tissue and muscle were lacerated.

Some segments of the small intestine perceive all through the abdominal wall (intestinal evisceration) by two openings located in the posteroventral abdominal region (skin wounds of $3 / 3 \mathrm{~cm}$ and $4 / 7 \mathrm{~cm}$ respectively); both orifices had hemorrhagically infiltrated edges. The eviscerated intestinal loops had a reddish serosa and ruptured mesentery. The internal exam of the body identified severe lung congestion, cerebral vasodilation and edema.

Final diagnosis was: polytraumatic lesions by biting, with associated evisceration and severe hemorrhages. The distribution of biting wounds in the dorsal and ventral parts of the body may suggest that attackers were most likely several of different sizes (i.e., tall and mean sized/small dogs).

\section{Case no. 4}

Examined subject and case history: dog body found in the wild environment (forest edge), Airedale Terrier, 5 years old male; no witnesses were declared. The corpse presents clayey soil in the hair coat and advanced cadaveric changes.

The external exam identified many variablesized wounds, most of them elongated with the opposite angular edges. In such areas, the underlying connective tissue and muscles were lacerated, with associated hemorrhages and hematomas. The skin wounds were identified in the following regions: head (frontal region, 1.8-1 $\mathrm{cm}$; Figure 7); the upper third of the left scapular region (two wounds of $2 / 1 \mathrm{~cm}$ and $3 / 1 \mathrm{~cm}$, respectively); the medial side of the elbow (Figure. 8 ) and in the lower third of the right hemithorax (in both locations $4 / 2 \mathrm{~cm}$ in size, Figure 9); the distal third of the neck $(1 / 0.5 \mathrm{~cm})$; the right ilium region $(0.5 / 0.5 \mathrm{~cm})$; the posterior side of the right leg $(0.5 / 0.5 \mathrm{~cm}$; Figure 10$)$; the sternal region $(0.5 / 0.5 \mathrm{~cm})$. The internal exam of the body identified severe post-hemorrhagic anemia, lung edema.
The final diagnosis was polytrauma by biting that lead to death. Aspect of the skin wounds and the laceration of the subcutaneous tissue/muscle (Figure 11), suggest they were produced most likely by dogs. Uneven distribution of the lesions (in the both dorsal and ventral regions of the body) could be due to mean to large dog aggressors.

\section{Results and discussions}

As observed in the cases described above, the biting wounds produced by canines had an angular and elongated aspect, features described by Munro and Munro (2008). Depending on the species involved, the topography of the lesions fluctuates. Accordingly, in deer the dogs attack the ventral or ventrolateral area of the neck and the lumbar or dorsal region of the prey. The biting wounds distribution in deer suggests the hunting instinct of the carnivores by attempting to suffocate the prey (Munro and Munro, 2008).

However, in the case of dog corpses, the distribution of biting wounds throughout the body is different. The most involved zones are the auricular area, limbs, sternum, flank region. It seems the biting wounds distribution is related to the size of the aggressor ( $\mathrm{dog}$ ) in relation to the victim. Regularly, medium-sized to small dogs attack lower parts of the prey whereas large dogs may induce wounds in the upper parts of the body (McKeirnan et al., 1984; Cowell and Penwic, 1989; Munro and Munro, 2008). Additionally, when dogs are part of a group, the "pack instinct" motivates to escalate the attack (Kneafsey and Condon, 1995; Santoro et al, 2011). The risk associated will be comparable to the number and size of dogs. If the prey is taller, attacking canids try to immobilize it by hitting at the buttocks and limbs; once the victim has been brought down, dogs will attack the prey until it stops moving (De Munnynck and Van de Voorde, 2002; Fonseca and Palacios, 2013).

As observed in the cases described, the biting wounds induced by canines are usually associated with the laceration of the subcutaneous tissue and related muscle, with or without the presence of skin wounds.

Biting wounds produced by canines should be differentiated by shutting wounds and stab (puncture) wounds. The shutting wounds are usually circular or slightly oval with minimal hemorrhage. Cartridge pellets or bullets generate difficult-to-follow traits and are often accompanied 


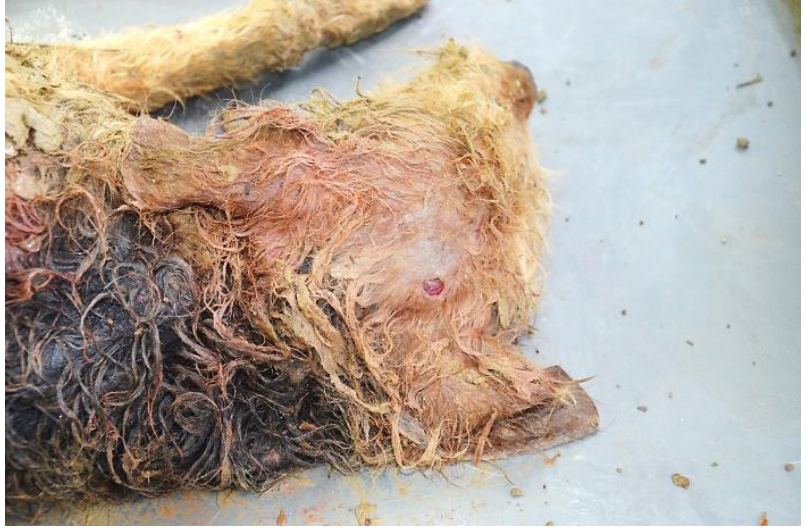

Figure 7. Bite wound in the frontal region (5 years old Airedale Terrier killed by dogs).

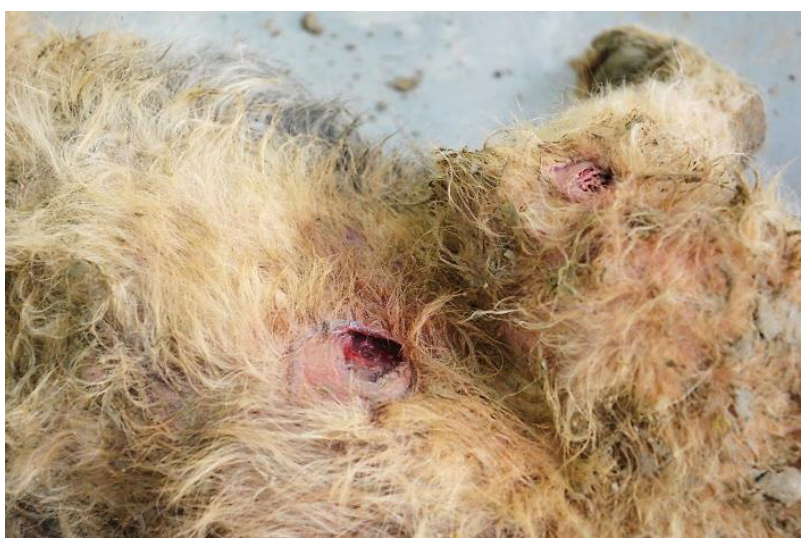

Figure 9. Laceration of the subcutaneous tissue; bite wound in the lower third of the right hemithorax.

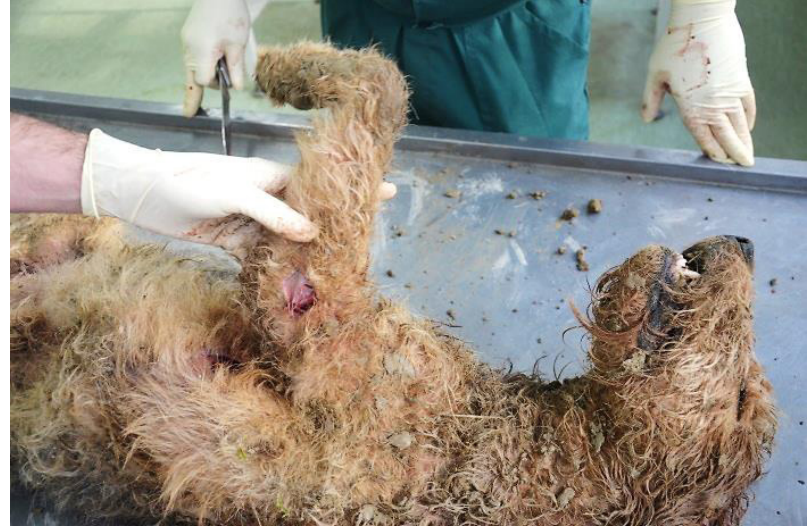

Figure 8. Angular aspect of the bite wound; the medial side of the elbow.



Figure 10. Uneven distribution of wounds throughout the body - the posterior side of the right leg.

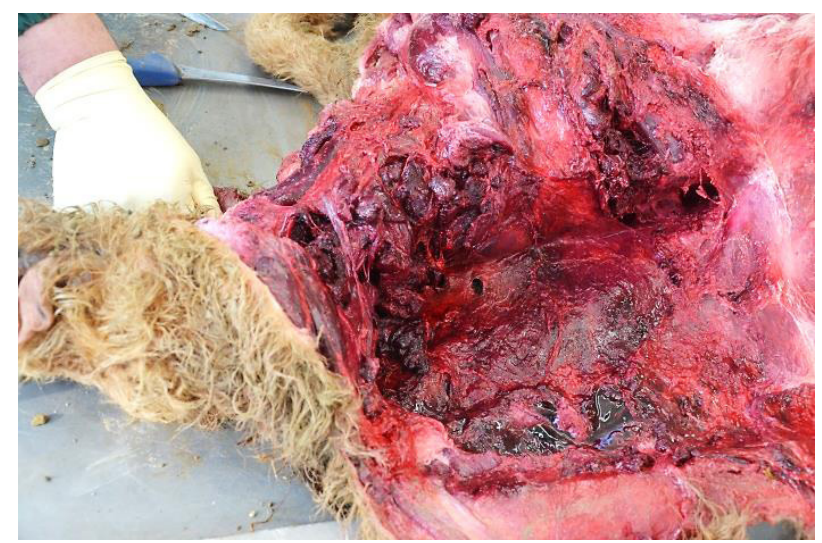

Figure 11. Laceration of the subcutaneous tissue and muscle (aspect following skinning).

by hemorrhagic lines through various tissues (Munro and Munro, 2008). Regarding the stab wounds, the exact shape of the penetrating object can infrequently be deduced from the outline of skin wound. A double-edged blade may leave a skin wound that tapers at both ends, comparing to the hole made by a single-edged blade. If the penetrating object is blunt, the entrance hole may 
show inversion of the margins (i.e., the skin was forced into the hole). Regarding the length of the stab wounds, it is usually significantly greater than the length of the skin wound. Overall, a specific weapon may cause wounds of various shapes and sizes depending on a number of circumstances, such as: the angle of attack, tightness and movement of the skin etc. Accordingly no confidence should be placed on the outline of a stab wound in the skin (Munro and Munro, 2008).

Shamir et al. (2002) described cases that suffered biting injuries in 185 dogs and 11 cats. A higher predisposition of male dogs was observed. Additionally, the most prevalent cases were represented by small dogs (less than $10 \mathrm{~kg}$ ) that were not only victims but suffered multiple, often deadly, injuries (Shamir et al., 2002). In a report from North America, the biting wounds distribution occurred (in a decreasing order of frequency) in legs, head and neck, thorax and abdomen regions (Holt and Griffin, 2000). In another report from Israel, the lesions occurred in some other body regions such as thorax, limbs, tail, head and neck. Such difference in lesion distribution corresponds to the prevalence of dog breeds in various geographic areas (Shamir et al., 2002).

The most common chest injuries that can be induced by dogs are: multiple costal fractures, often with a circular aspect that communicate with the chest cavity; perforation of pulmonary parenchyma and hemotorax. Abdominal lesions are characterized by the presence of hematomas and abdominal wall bleeding, perforation or laceration of the abdominal muscles, ruptures of abdominal parenchymal organs with massive intra-abdominal hemorrhage. Other lesions that may occur following dog bite are fractures at different levels of the spine, fractures of the pelvic bones and the skull (McKeirnan et al., 1984; Cowell and Penwic, 1989; Munro and Munro, 2008).

There are many more papers describing dog attacks on humans. In such scenario, the injuries are represented by particular punctiform " $\mathrm{V}$ "shaped holes and lesions presenting irregular and furrowed borders. The abovementioned typical "hole-and-a-tear" is a puncture wound (the round hole) produced by the canine tooth of either the maxilla or mandible on one side, which serves as an anchorage point, followed by shaking of the head creating tears enhanced by the cadaver's body weight (Fonseca et al., 2015). Wounds are licked in many cases and may take on a very bright and polished aspect (Tsokos et al., 2007; Colard et al., 2015). This pattern is accompanied by skin abrasions and superficial linear abrasions arranged parallel corresponding to claw marks, and usually present in the contour of the bite (Tsokos et al., 2007; Santoro et al., 2011; Fonseca and Palacios, 2013). There is a paucity of literature on the individualization of dog bite marks, discriminating bite marks from claw marks and their procedures (Bernitz et al., 2012; Fonseca et al., 2015).

\section{Conclusion}

Investigation of bite-marks is an evolving field of forensic veterinary science and remains a provocation for veterinary pathologists. Injuries caused to animals, whether accidental or deliberate, include a variety of situations that are sporadically presented worldwide. All analyzed cases displayed numerous usually symmetrical (opposed) wounds, with angular margins and elongated aspect. Additionally, the laceration of the subcutaneous tissue and related muscle was observed, all features described being typical for wounds produced by canines. Depending on the species involved, the topography of lesions varied. In deer, the ventrolateral area of the neck and the lumbar/dorsal regions were more commonly affected. In dog corpses, the lesions were mainly located in the auricular region, limbs, sternal region and abdominal region. Finally, other similar studies are required to have a better description for a number of circumstances.

Acknowledgments. This research did not receive any specific grant from funding agencies in the public, commercial, or not-for-profit sectors.

\section{References}

1. Bernitz H, Bernitz Z, Steenkamp G, Blumenthal R, Stols G (2012). The individualisation of a dog bite mark: a case study highlighting the bite mark analysis, with emphasis on differences between dog and human bite marks. Int J Legal Med 126(3): 441-446.

2. Colard T, Delannoy Y, Naji S, Gosset D, Hartnett K, Bécart A (2015). Specific patterns of canine scavenging in indoor settings. J Forensic Sci 60(2):495-500.

3. Cowell AK, Penwic RC (1989). Dog bite wounds: a study of 93 cases. Compend Contin Educ Pract Vet 11: 313-320. 
4. De Munnynck K, Van de Voorde W (2002). Forensic approach of fatal dog attacks: a case report and literature review. Int J Legal Med 116(5):295-300.

5. Fonseca GM, Palacios R (2013). An unusual case of predation: dog pack or cougar attack? J Forensic Sci 58(1):224-227.

6. Fonseca G, Mora E, Lucena J, Cantin M (2015). Forensic studies of dog attacks on humans: a focus on bite mark analysis. Research and Reports in Forensic Medical Science 5:39-51.

7. Holt DE, Griffin G (2000). Bite wounds in dogs and cats. Vet Clin North Am Small Anim Pract, 669-679.

8. Kneafsey B, Condon KC (1995). Severe dog-bite injuries, introducing the concept of pack attack: a literature review and seven case reports. Injury 26(1): 37-41.
9. Mckeirnan BC, Adams WM, Huse DC (1984). Thoracic bite wounds and associated internal injury in 11 dogs and 1 cat. J Am Vet Med Assoc 184:959-964.

10. Munro R, Munro MC Helen (2008). Animal Abuse and Unlawful Killing. Saunders Elsevier, 1-98.

11. Santoro V, Smaldone G, Lozito P, Smaldone M, Introna F (2011). A forensic approach to fatal dog attacks. A case study and review of the literature. Forensic Sci Int 206(13):e37-e42.

12. Shamir MH, Leisner S, Klement E, Gonen E, Johnston D (2002). Dog bite wounds in dogs and cats: a retrospective study of 196 cases. J Vet Med A 49:107-112.

13. Tsokos M, Byard RW, Püschel K (2007). Extensive and mutilating craniofacial trauma involving defleshing and decapitation: unusual features of fatal dog attacks in the young. Am J Forensic Med Pathol 28(2):131-136. 